\title{
Depression Screenings during Routine Visits in a Reproductive Healthcare Setting: Identifying Depressive Symptoms in African American Adolescent Males
}

\author{
Kenia Johnson $^{1 *}$, Karia Kelch-Oliver ${ }^{2}$, Chaundrissa Oyeshiku Smith ${ }^{3}$, \\ Sophia Edukere Green ${ }^{3}$, Triphinia M. Wallace ${ }^{4}$, Melissa Kottke ${ }^{3}$, Marietta H. Collins ${ }^{3}$ \\ ${ }^{1}$ Department of Social Sciences, Chattahoochee Technical College, Marietta, GA, USA \\ ${ }^{2}$ Morehouse School of Medicine, Atlanta, GA, USA \\ ${ }^{3}$ Emory University School of Medicine, Atlanta, GA, USA \\ ${ }^{4}$ Community Counseling Solutions, East Point, GA, USA \\ Email: "kenia.johnson@chattahoocheetech.edu
}

Received July $11^{\text {th }}$, 2012; revised August $13^{\text {th }}$, 2012; accepted September $15^{\text {th }}, 2012$

\begin{abstract}
Depression is a disorder which affects many youth, and only one third of adolescents receive mental health treatment for their depression. Yet, approximately $90 \%$ of adolescents visit their primary care providers on average 2 - 3 times per year. This number suggests the important role that primary care settings can play regarding the early diagnosis and treatment of depression during adolescence. This paper presents findings of clinically significant depressive symptoms in African American male adolescents receiving routine health care services within an adolescent reproductive health clinic. The adolescent reproductive health clinic is housed within a large urban, university-affiliated teaching hospital. 49 African American male adolescents (ages 13 to 19) completed the Center for Epidemiologic Studies Depression Scale (CES-D) (Radloff, 1977), a brief depression screening questionnaire, as a part of their clinic visit. Results revealed higher rates of depressive symptoms in this subsample of African American male adolescents when compared to estimated prevalence rates of depression for adolescents as reported by large-scale studies and meta-analysis data. This supports the notion that primary and reproductive healthcare settings are viable settings for the identification of depressive symptoms, particularly among low-income, African American male adolescents. Risk factors, symptom presentation, and mental health stigma associated with this population are discussed. Psychosocial interventions and recommendations for the integration of primary healthcare and behavioral health consultation services are presented.
\end{abstract}

Keywords: Adolescents; Depressive Symptoms; African American Males; Reproductive Health Clinics; Primary Care

\section{Adolescent Depression}

Depression in children and adolescents was scarcely recognized in the empirical literature until the 1970s (Angold, Worthmon, \& Costello, 2003), but has now become a major public health concern (Cote et al., 2003) due in part to large-scale studies reporting that up to $3 \%$ of children and $8 \%$ of adolescents suffer from depression (US Department of Health and Human Services, 2001). The prevalence rates of depression are similar for males and females before puberty, but after puberty depression rates increase, especially in females (Angold et al., 2003). Comparatively, female adolescents have a 2 to 3 times higher prevalence rate of major depressive episode $(12 \%-13 \%)$, whereas the prevalence rate of major depressive episode for male adolescents is approximately $4 \%$ to 6\% (Substance Abuse and Mental Health Services Administration Office of Applied Studies, 2008). Overall, based on metaanalytic data, the prevalence of depression estimates for adolescents ages 13 to 18 is approximately 5\% to 15\% (Costello, Erkanli, \& Angold, 2006). Adolescents who are depressed are

*Corresponding author. at least three times more likely than non-depressed adolescents to remain depressed during young adulthood (Cardemil, Reivich, \& Seligman, 2002; Cote et al., 2003; Kessler et al., 2001). Moreover, depressed teens are more likely to drop out of school, engage in substance abuse, and become teen parents compared to non-depressed teens (Horowitz \& Garber, 2006; US Department of Health and Human Services, 2008). Unfortunately, only about one third of adolescents receive professional help for their depression, despite such high prevalence rates of adolescent depression (Kessler et al., 2001; Stein, Zitner, \& Jensen, 2006; Wu et al., 2001). Therefore, early identification and treatment of adolescent depression is crucial in order to prevent more detrimental psychosocial outcomes later in life.

\section{Racial/Ethnic and Socioeconomic Differences in Depression}

Generally, depressive symptoms in low-income, urban African American adolescents are largely understudied (Shaffer, Forehand, \& Kotchick, 2002). Although research remains limited, it has been suggested that racial/ethnic minority adoles- 
cents may be at an elevated risk for depression (Cardemil et al., 2002; Rushton, Forcier, \& Schectman, 2002; Shaffer et al., 2002). Reviewing the adult literature, some research suggests higher rates of depression in African Americans compared to Caucasians (Wight, Aneshensel, Botticello, \& Sepúlveda, 2005). However, overall results appear inconsistent within the adult literature, with other studies reporting lower (Angold et al., 2002; Riolo, Nguyen, Greden, \& King, 2005) or equivalent rates of depression between African American and Caucasian individuals (Kessler et al., 2003; Shaffer et al., 2002). In a study among a sample of African American adolescent females, Collins et al. (2010) found that clinically significant depressive symptoms were endorsed at rates higher than the general population. Research has also indicated that depressive symptom presentation may manifest differently across racial and ethnic groups (Cardemil et al., 2002; Shaffer et al., 2002). African American adolescents have reported more symptoms of diminished pleasure, increased anger, aggression, and irritability than Caucasian, Hispanic, and Asian American adolescents (Choi \& Gi Park, 2006). Varied depressive symptom presentation in African American youth may mask the symptoms, making it less recognizable to health care professionals, further complicating accurate assessment and diagnosis.

Additional studies of adolescent depression which include racial and ethnic minorities indicate that poverty, low socioeconomic status, and lack of academic and educational attainment are significant risk factors for depression (Anderson \& Mayes, 2010; Cardemil et al., 2002; Riolo et al., 2005). A substantially higher proportion of racial and ethnic minority youth are among the economically disadvantaged, which creates more stressful environmental risk factors (Anderson \& Mayes, 2010). Thus, urban, low-income African American adolescents living in high-risk environments (i.e., characterized by crime and violence, poverty, substance abuse) may experience depression at higher levels than adolescents from other racial/ethnic groups in the US (Cardemil et al., 2002; Lindsey, Joe, \& Nebbitt, 2010; US Department of Health and Human Services, 2001). African American adolescents, especially males, may be more susceptible due to low social support networks, greater exposure to delinquent peers and violence, the perception of fewer future opportunities, and other risks associated with living in high-risk environments (Hammack, Richards, Luo, Edlynn, \& Roy, 2004; Lindsey et al., 2010; Nebbitt \& Lombe, 2008). Therefore, identification of mechanisms to improve early detection of depressive symptoms among African American male adolescents warrants further attention.

\section{Perceived Stigma and Outcomes of Untreated Depression}

Differences by race/ethnicity in help-seeking, accessing mental health and using psychotropic medications may also account for differences in prevalence rates among African American adolescents, particularly African American adolescent males (Riolo et al., 2005). African American adolescents are reported to be less likely to seek and receive treatment for depression compared to their Caucasian counterparts (Riolo et al., 2005; US Department of Health and Human Services, 2001). The SAMSHA report revealed that in 2008, 9.5\% of African American adolescents, as compared to $12.7 \%$ of Caucasian adolescents, utilized outpatient mental health services (Substance Abuse and Mental Health Services Administration Of- fice of Applied Studies, 2008). The underutilization of formal mental health services among African American adolescents with depression has spawned studies designed to examine factors that underlie symptom and help-seeking behaviors (Lindsey et al., 2010). Explanations for the observed lower rates of mental health use for this group of individuals include distrust of mental health professionals because of historical abuses relating back to slavery and a perceived lack of cultural sensitivity by providers (Goldston et al., 2008). Additionally, institutional barriers to mental health care, such as the absence of or inadequate health insurance and the prevalence of culturally inappropriate screening measures, diagnostic procedures, and treatment modalities exist that diminish help-seeking and treatment compliant behaviors (Atdjian \& Vega, 2005; Copeland, 2006).

Prior studies have shown that African American adolescents, especially African American adolescent males who experience depression, may be particularly sensitive to stigma associated with depression and mental health treatment (Lindsey et al., 2010). In their examination of mental health stigma, social support, and depressive symptoms in African American males, Lindsey et al. (2010) showed an association between participants' use of mental health professionals and feeling "shamed." Furthermore, Lindsey et al. (2010) suggested that the under-utilization of mental health services by African American males was related to feelings of being stigmatized by their peers. African American male adolescents tend to view the acknowledgement of depressive feelings as culturally incongruent (Molock et al., 2007; Moses, 2009), and thus will typically talk to family and friends prior to and/or in lieu of seeking mental health services (Lindsey et al., 2010).

Additionally, suicidal ideation and suicide, which could be an outcome of untreated depression and depressive symptoms, is the third leading cause of death among adolescents (Anderson, 2002; Franko et al., 2005; Mazzaferro et al., 2006; Rudolph, 2008). Although women have been documented to make more suicide attempts, American males of all ethnicities continue to be more effective at ending their lives (Centers for Disease Control and Prevention National Center for Injury Prevention and Control). According to the 2007 United States Census Bureau, it is reported that American males of all ages completed suicide at a rate of 18.4 out of every 100,000 while their female counterparts completed suicide at a much lower rate of 4.7 per 100,000 . This trend was shown to be similar among African Americans, with higher suicide rates for African American males of all ages $(8.8$ per 100,000$)$ compared to the suicide rates for African American females of all ages (1.7 per 100,000) (Centers for Disease Control and Prevention National Center for Injury Prevention and Control). Recent reports indicate observing the highest suicide completion risk among 25 44 year old individuals (13.7 per 100,000) and among 15 - 24 year old African American males (10.3 per 100,000) (Centers for Disease Control and Prevention National Center for Injury Prevention and Control).

However, this trend may not provide an accurate representation of this phenomenon within the African American adolescent culture. Goldston et al. (2008), reported that for some depressed youth, stigma and the importance of not appearing vulnerable results in "precipitated" suicide, which refers to instance in which individuals try to provoke others into killing them, thereby the individual seems to be a victim of a homicide. Cases of precipitated suicide are disproportionately present in 
African American males and may be viewed as more culturally appropriate means to ending their lives (Gibbs, 1988). Clinical factors such as depression, hopelessness, and poor problemsolving skills have been identified as significant contributors to suicide and suicidal behaviors (Boyd \& Foley, 2009).

\section{Integration of Mental Health and Primary Care in Identifying Adolescent Depressive Symptoms}

Primary care providers (PCPs) (i.e., providers in family medicine, pediatrics, obstetrics/gynecology, or adolescent medicine) are often the first (and sometimes only) point of contact individuals have with the health care system (Nimalasuriya, Compton, \& Guillory, 2009). Racial/ethnic minority and lowincome adolescents have been shown to access primary care clinics more often than mental health clinics (Stein et al., 2006). Within research protocols, mental health problems have been identified in $8 \%$ to $13 \%$ of adolescents attending routine physical examinations in pediatric practice (Husky, Miller, McGuire, Flynn, \& Olfson, 2011). African Americans, in particular, are more likely to receive mental health care from a PCP than from a mental health specialist (Gallo, Bogner, Morales, \& Ford, 2005; Stockdale, Lagomasino, Siddique, McGuire, \& Miranda, 2008). Approximately, $90 \%$ of adolescents visit their primary care providers on average 2 to 3 times per year (Stein et al., 2006). These statistics indicate that there is an opportunity for primary care providers to provide universal screening of depression, and in doing so, potentially prevent suicide among a significant number of adolescents (Frankenfield et al., 2000; Kramer, Beaudin, \& Thrush, 2005); particularly in those groups that are at-risk for depressive symptoms (e.g., low-income, racial/ethnic minority adolescents).

Within primary care settings, pediatricians are often de facto providers of mental health care to young people, with the unique opportunity for the universal screening, identification, and treatment of a significant percentage of adolescents at risk for depression (Husky et al., 2011). The US Preventive Services Task Force (USPSTF) recommends that all primary care providers should have "systems in place, whether within the primary care setting itself (i.e., on-site) or through collaborations with mental health professionals, to ensure the accurate diagnosis, effective treatment, and follow-up of depression" (US Preventive Services Task Force, 2009). It is further recommended by the USPSTF that primary care providers screen adolescents for depression annually from 12 through 18 years of age during routine visits (Hamrin \& Magorno, 2010). Several obstacles appear to impinge on a PCP's ability to screen for mental health concerns. Prior studies of pediatric, adolescent medicine, and family physicians have indicated reports that time constraints related to medical productivity requirements, limited/no reimbursement for mental health services, lack/ limitation of skills need to diagnosis and effectively treat mental health needs, and limited/no treatment referrals all represent challenges faced by PCPs in integrating mental health assessment into their practice (Hacker et al., 2006; Zuckerbrot et al., 2007).

Yet, there is evidence among studies conducted in the adult depression literature which demonstrate the effectiveness of collaborative care between mental health and primary care (Zuckerbrot, Cheung, Jensen, Stein, \& Laraque, 2007). Moreover, an integrated model of collaborative care is considered to be especially important for racial/ethnic minority and/or low- income adolescents who may have limited access to healthcare services. The use of standardized screening tools in pediatric practice (e.g., Beck Depression Inventory-Primary Care Version, Patient Health Questionnaire for Adolescents) have been shown as an effective method to improve detection rates of depression and other psychosocial issues during routine primary care visits (Johnson, Harris, Spitzer, \& Williams, 2002; Winter, Steer, Jones-Hicks, \& Beck, 1999). For example, in a sample of patients aged 4 years 11 months to 19 years of age, Hacker et al. (2006) implemented the Pediatric Symptom Checklist (35-item) to identify mental health problems during an annual screening at an urban pediatric setting. Results revealed that $6 \%$ of the patient population endorsed positive Pediatric Symptom Checklist scores, which indicated that the child had emotional or behavioral problems that warranted clinical attention. The authors concluded that mental health screenings can be effectively implemented within a pediatric clinic setting. Similarly, Collins et al. (2010) incorporated a brief depression screening tool, The Center for Epidemiologic Studies Depression Scale (CES-D), as a part of the routine clinical procedures within an urban reproductive health clinic. The patient population included African American adolescent females between ages 12 and 19 years. The CES-D was able to identify approximately $19 \%$ of adolescent females presenting with clinically significant levels of depressive symptoms.

Zuckerbrot, Maxon, Pagar et al., (2007) implemented the administration a 22-item paper-and-pencil survey (i.e., Columbia Depression Scale, CDS) to all eligible patients. The results revealed that the average completion time of the paper-andpencil survey was 4.6 minutes, and appeared to be well perceived, had minimal staff resistance, and was accepted among parents, patients, and providers. Therefore, Zuckerbrot et al. (2007) concluded that brief universal systematic depression screenings can be successfully implemented within pediatric clinics. Despite this finding, a limited number of PCPs have utilized standardized assessment methods to screen for depressive symptoms in their adolescent patient population (Zuckerbrot \& Jensen, 2006) suggesting that cases of depression are being missed, which could result in detrimental mental health trajectories, particularly among low-income African American male adolescents, given the purported vulnerability and limited access of mental health clinics among this population.

\section{Statement of the Problem}

This paper implemented a standardized depression screening tool and examined the detection rates of depressive symptoms in primarily low-income, African American male adolescents who receive routine reproductive health care services in an adolescent reproductive health clinic located within an urban hospital. It was expected that integrating a depression screening tool during a routine physical examination would identify clinically significant depressive symptoms within a population that generally would not self-identify or receive mental health treatment. As previously stated, the rate of depression for adolescents in the general population is estimated to be between $5 \%$ and $15 \%$. However, there are numerous environmental experiences associated with this population of low-income, African American male adolescents such as socioeconomic stress, exposure to trauma, difference in symptom presentation, and the stigma of mental health. Therefore, given these variables, it was hypothesized that the use of an instrument assess- 
ing depression within a primary care adolescent reproductive health clinic would reveal endorsements of clinically significant depressive symptoms among African American adolescent males at this clinic. It was further expected that, within this sample, depressive symptoms would be endorsed at an equal or greater rate than that of the general adolescent population.

\section{Method}

Note: This research was an outgrowth of another study which identified clinically significant depressive symptoms in African American females in an urban reproductive health clinic (Collins et al., 2010). Therefore the methodological issues are the same as the abovementioned study.

\section{Participants}

Participants included 49 African American adolescent males between ages 13 and 19 years $(M=17.61, S D=1.35)$ that attended an appointment at a large, university-affiliated, public hospital-based adolescent primary care reproductive health clinic in the southeastern region of the United States. All adolescent patients requesting services at the clinic completed a depression screening questionnaire. The depression screening questionnaire was included in the Adolescent Reproductive Health Clinic's routine clinic intake packet. Thus, the data were collected as part of routine clinical procedures, as the intake and all depression symptom questionnaires completed during the clinic visit are included in the study results. Only completed depression screening questionnaires were used in the final sample. Approval for the inclusion of the depression screening questionnaire was obtained from the university-affiliated Institutional Review Board.

\section{Setting}

The hospital-based adolescent primary care reproductive health clinic is housed within a large urban, university affiliated teaching hospital and provides an array of services to primarily African American, low income adolescent males and females. The clinic typically accommodates nearly 2000 female and male adolescent clinic visits annually, of which $20 \%$ are males. The average age of patients served in the clinic is 17 years old (range 11 - 20 years old), and $92 \%$ of patients describe themselves as African American. Services provided in the clinic include: pregnancy tests and prevention, testing and treatment for sexually transmitted infections (STIs), contraceptives, routine gynecological and postpartum examinations, sports physicals, pregnancy prevention educational sessions for parents and adolescents utilizing a postponing sexual involvement curriculum, case management, and psychological counseling. STI diagnosis and treatment and pregnancy tests are the most frequently used services. Staff includes medical providers/clinicians (medical doctors, medical interns/residents, physician assistants, nurses), health educators, and psychology trainees (psychology interns and postdoctoral fellows). Psychology trainees are available to provide mental health consultation to adolescents referred by staff and receive consultation and weekly supervision from a licensed clinical psychologist who is on-call for any mental health emergency. Approximately 20\% of all adolescents served at the clinic are referred to psychology trainees for mental health consultation. Although adolescent reproductive health clinics exist nationally and worldwide, the inclusion of mental health services, an individualized case management approach using health educators, peer educators/ teen leaders, a computer center, and the clinic's partnership with the local school system make this clinic particularly unique.

\section{Procedures}

Adolescents in the sample completed a brief depression screening questionnaire (e.g., Center for Epidemiologic Studies Depression Scale (CES-D), Radloff, 1977) as part of a routine clinic visit. During check-in at the clinic, adolescents were provided with a consent form explaining the purpose of the study and requesting permission to obtain a copy of their responses on the questionnaire for research. Adolescents are considered "emancipated" for reproductive health care services within the state where the study was conducted; therefore, parental consent was not required for clinic services. Parental consent was not required for the depression screening measure because it was considered to be a routine part of the ongoing intake procedures within the clinic. Adolescents were provided with the clinic's routine intake packet. The CES-D form was one of several forms in the intake packet that all adolescents requesting services in the clinic completed prior to being seen by the medical provider/clinician. Adolescents also responded to an additional item that assessed for suicidal ideation (SI). Adolescents who obtained a total score of 16 or above on the depression screening tool and/or endorsed SI on the clinic form were referred to a psychology trainee (psychology postdoctoral fellow or intern) and provided with a brief psychosocial assessment (i.e. mental health screening) by the psychology trainee as part of their routine visit to the reproductive health clinic. Adolescents who endorsed SI were assessed by the psychology trainee for suicidal intent and plan, and if the adolescent was determined to be suicidal, referral for psychiatric hospitalization was made. As needed, adolescents were provided with ongoing mental health services after parental consent was obtained for psychological services.

\section{Measures}

The Center for Epidemiologic Studies Depression Scale (CES-D) (Radloff, 1977) is a 20-item self-report depression screening measure. Participants were asked to respond to the items based on their feelings within the past week. Items are rated on a four-point scale, with $0=$ rarely or none of the time (less than 1 day), 1 = some or a little of the time ( 1 - 2 days), 2 = occasionally or a moderate amount of time (3 - 4 days), and 4 $=$ most or all of the time ( $5-7$ days). The CES-D scores range from 0 to 60, with higher scores indicating more severe depressive symptoms. Consistent with previous studies, a total score of 16 or greater is indicative of adolescents with clinically significant depressive symptoms. Furthermore, Munoz (2005) offered the following guide for interpreting CES-D scores, which will be applied in the analysis of the current study: average range of depressed symptoms (scores less than 16), borderline elevation of depressed symptoms (scores 16 to 24), and significant elevation of depressed symptoms (scores 24 and above). The CES-D has been used effectively with adolescents, and reliability and validity have been tested and established in African American populations (Radloff, 1991). The CES-D is 
shown to be valid and to have good test-retest reliability (.77 - .92). Within the current sample, internal consistency for the CES-D was .85. One item was added to the CES-D in the current study that was not included in the total CES-D score. Participants were asked to respond to an item regarding their experience of suicidal thoughts within the past week, "I felt that I would be better off dead or had thoughts of hurting myself”.

\section{Results}

The total number of depressive symptoms endorsed on the CES-D ranged from 0 to $36(M=8.92, S D=8.19)$. A Spearman's rank correlation analysis revealed that there was not a significant relationship between age and total number of depressive symptoms endorsed on the CES-D. However, approximately $18 \%(n=9)$ of the overall sample of $n=49$ reported significant levels of depressive symptoms on the CES-D (score of 16 or higher) with a mean score of 23.67 (SD $=6.28$ ). Within the subsample of participants with CES-D scores higher than 16 , approximately $67 \%(n=6)$ of the subsample endorsed borderline elevation of depressive symptoms (CES-D score between 16 and 24) with a mean score of 20.17 ( $S D=2.64)$, and approximately $33 \%(n=3)$ of the subsample reported significant depressive symptoms indicative of a clinical diagnosis of depression (CES-D score above 24) with a mean score of $30.67(S D=5.51)$. The reported rates of depressive symptoms within the overall sample and subsample of African American male adolescents in the present study are higher than the estimated prevalence rates of depression reported for adolescents ages 13 - 18 (5\% - 15\%) (Costello et al., 2006). Within the subsample of participants with CES-D scores higher than 16, correlation analysis was performed to determine the relationship between age and endorsement of significant levels of depressive symptoms on the CES-D, and did not find any significant associations. Additionally, approximately 6\% $(n=3)$ of the total sample endorsed the additional item indicating experience of suicidal thoughts within the past week. Table 1 displays descriptive information including means and standard deviation, for the subsample of participants whose total CES-D scores were higher than the published cutoff (CES-D scores above 16).

\section{Implications/Discussion}

The purpose of this study was to demonstrate the clinical utility of implementing a brief mental health screening measure within a reproductive health care setting to support identification of depressive symptoms among a low-income, African American male adolescent population. In this study, we were able to support our hypothesis with our major finding that the inclusion of the CES-D resulted in approximately $18 \%$ of African American male adolescents being successfully identified as experiencing clinically significant depressive symptoms which are higher than the estimated prevalence rates of depression reported for adolescents ages 13 to 18 years old (5\% - 15\%). Given the relatively small overall sample size $(n=49)$, this finding is noteworthy as it also supports previous research reporting the identification of mental health problems in $8 \%$ to $13 \%$ of adolescents attending routine physical examinations (Husky, Miller, McGuire, Flynn, \& Olfson, 2011). Additionally, the results of the current study are consistent with results from the Collins et al. study (2010) in which approximately 19\% of African American females accessing primary care in a reproductive health clinic were identified as experiencing significant depressive symptoms using the CES-D. The results across this study and the Collins et al. study (2010) may offer broader implications for identifying and understanding depression within the general population of African American youth. As previously mentioned, research has indicated varied depression symptom presentation across racial and ethnic groups (Cardemil et al., 2002; Shaffer et al., 2002). In particular, the literature has noted increased reports of symptoms of diminished pleasure, increased anger, aggression, and irritability among African American adolescents than Caucasian, Hispanic, and Asian American adolescents (Choi \& Gi Park, 2006). The use of the CES-D with African American adolescents may serve as a first-stage screening device that allows for the identification of depressive mood that can develop into a depressive episode or another form of serious depression should it continue for long.

Furthermore, although it was not statistically significant, it is important to note that three participants within this small sample indicated experiencing suicidal thoughts within the past week. Thus, the addition of the item inquiring about suicidal ideation to the CES-D in the current study seemed to give providers an assessment of multiple risk factors during a routine visit. Moreover, the design of the study allowed for the immediate referral, consultation, and follow-up by a mental health trainee/professional when significant depressive symptoms or suicidal ideation were endorsed. These findings support the assertion that primary care and reproductive health settings, particularly those that serve low-income and/or at-risk racial/ ethnic minority adolescents, can successfully and feasibly screen patients for mental health issues as a part of routine medical care. These results also indicate that screening for depressive symptoms in African American adolescent males in primary care and reproductive health clinics is effective in identifying a population of youth that generally may not selfidentify themselves as being in need of mental health treatment.

Table 1.

Elevated depression scores report on the CES-D among the subsample $(n=9)$.

\begin{tabular}{ccccc}
\hline Age in years & Frequency $(n=9)$ & Mean & $S D$ & Range of CES-D Scores \\
\hline 13 & - & - & - & - \\
14 & - & - & - & - \\
16 & 1 & - & - & 21 \\
17 & 2 & 30.00 & 8.49 & $24-36$ \\
18 & 4 & 21.25 & 6.55 & $17-31$ \\
19 & 2 & 23.50 & 2.12 & $22-25$ \\
\hline
\end{tabular}


Additionally, this study supports research that suggest that screening for suicide risk factors is possible and more likely to occur in conjunction with mental health screenings that are perceived to cause little disruption to routine clinic procedures (Frankenfield et al., 2000).

It is important to consider the limitations of the findings of this study. First, it is likely that the results were unable to detect significant group differences between age and depressive symptoms given the small sample size, and still disproportionate number of participants representing each group. Given the relative homogeneity of this sample with regard to race, gender, and socioeconomic status, it is likely that generalizeability is limited. Furthermore, the CES-D is a measure of global distress related to depressive symptoms, which unfortunately did not account for further investigation of subcategories of depression such as somatic, neurovegetative, or mood/cognitive symptoms which may have yielded different results. Finally, this study relied solely on self-report of depressive symptoms, and the results may be limited by the participants' potential tendency to present in a socially desirable manner, thus minimizing depressive symptoms. Collaborative information (i.e., objective, teacher and/or parental report) regarding the participants' experience of depression were not obtained or incorporated.

\section{Recommendations}

It is common for adolescent males present to clinics with chief complaints unrelated to depression, such as sports-related injuries or difficulties with a chronic condition such as asthma, dermatological problems such as acne, or acute infections such as strep throat (Westwood \& Pinzon, 2008). As previously mentioned, in the case of African American male adolescents, it is possible that cultural stigma associated with depression as well as gender socialization may lead to differences in depression symptom presentation which are more socially acceptable. Survey reports reveal that roughly $20 \%$ or less of adolescents seen in primary health clinics or pediatric emergency medicine are actually screened for mental health issues (Husky et al., 2011). Yet, by the time adolescents reach the age of 16-year-old, it has been estimated that as many as approximately $37 \%$ of adolescents will meet criteria for at least one mental disorder (Husky et al., 2011). Thus, it is important for brief mental health screenings to be integrated into primary care practices to improve identification of mental health difficulties among adolescents. Furthermore, universal screening of depressive symptoms that are sensitive to the differences within the African American male adolescent population is especially essential.

Demonstrating the feasibility of screening for depressive symptoms in adolescents is only a start to addressing this issue. Consultation, referral, and treatment of these symptoms are an important aspect of adolescent health and of this research. In the case of adolescent depression, in which prevalence rates increase with age, some adolescents may present to primary care and reproductive health clinics with clinically significant depression. However, others may present with less severe subclinical forms of depression (i.e., depressive symptoms) that may be more common in this population of African American male adolescents, and also require clinical attention. Consistent with the recommendation for systematic screening and the integration of biological and psychological treatments that includes diagnosis, psychotherapy, and follow up (US Preventive Services Task Force, 2009), the clinic within the current study provided multiple services entailing sports physicals, case management, psychological consultation, and ongoing mental health services as needed (with parental consent). A unique feature to this study was the inclusion of mental health professionals within the primary clinic, which has been noted as an effective strategy in encouraging appropriate psychosocial treatment for adolescents experiencing mental health difficulties (Bower, Garralda, Kramer, Harrington, \& Sibbald, 2001; Kelleher, Campo, \& Gardner, 2006). However, within settings in which staff may not include mental professionals, empirical studies on the efficacy of primary care-initiated psychosocial intervention for adolescents have shown promising results (Asarnow et al., 2005; Johnson et al., 2002; Walker et al., 2002). The types of psychosocial interventions offered by primary care clinicians may include counseling (e.g., reflective listening, problem solving, cognitive reframing, psychoeducation via literature or information), prescribing psychotropic medication, or a referral to specialty care mental health clinic (Williams, Klinepeter, Palmes, Pulley, \& Foy, 2004; Williams, O’Connor, Eder, \& Whitlock, 2009). Also, if on-site mental health consultation is not feasible, primary care and reproductive health settings should be encouraged to establish relationships with mental health professionals in the community for consultation and referral of adolescents with depressive symptoms. As research continues in this area, it will be essential that it focuses not only on identifying feasible screening mechanisms for identifying depression in primary care settings, but also identifying practical applications of psychosocial interventions to treat those who are identified with depressive symptoms within primary care settings.

\section{Conclusion}

Results of the current study are in support of the integration of routine, universal depression screening and subsequent referral to mental health professions within primary care and reproductive health settings. It is hoped that the results of the current study contribute to an increased awareness of the need for early identification and treatment of underserved African American adolescent males with depressive symptoms. Despite the fact that depression during adolescence is a major public health concern with potential long term consequences, it often is unrecognized, under-diagnosed, and/or untreated. Given the public health concern of adolescent depression, the stigma of mental health in some communities, as well as the likelihood that most depressed adolescents do not receive mental health services, it is imperative that primary care and reproductive health settings consider incorporating a brief depression screening measure in their routine practices. Additionally, consultation and referral for mental health treatment for adolescents with depressive symptoms will facilitate the delivery of behavioral health services and lead to meaningful improvements in adolescent care.

\section{REFERENCES}

Anderson, E. R., \& Mayes, L. C. (2010). Race/ethnicity and internalizing disorders in youth: A review. Clinical Psychology Review, 30, 338-348. doi:10.1016/j.cpr.2009.12.008

Anderson, R. (2002). Deaths: Leading causes for 2000 (Vol. 50). National Vital Statistics Reports. Hyattsville, MD: Centers for Disease Control and Prevention, National Center for Health Statistics, Na- 
tional Vital Statistics System.

Angold, A., Erkanli, A., Farmer, E., Fairbank, J., Burns, B., Keeler, G., \& Costello, E. (2002). Psychiatric disorder, impairment, and service use in rural African American and white youth. Archives of General Psychiatry, 59, 893-901. doi:10.1001/archpsyc.59.10.893

Angold, A., Worthmon, C. M., \& Costello, E. J. (2003). Gender differences at puberty. In C. Hayward (Ed.), Puberty and depression. New York: Cambridge University Press.

Asarnow, J., Jaycox, L., Duan, N., LaBorde, A., Rea, M., Murray, P., Anderson, M., Landon, C., Tang, L., \& Wells, K. (2005). Effectiveness of a quality improvement intervention for adolescent depression in primary care clinics a randomized controlled trial. Journal of the American Medical Association, 293, 311-319. doi:10.1001/jama.293.3.311

Atdjian, S., \& Vega, W. A. (2005). Disparities in mental health treatment in US racial and ethnic minority groups: Implications for psychiatrists. Psychiatric Services, 56, 1600-1602. doi:10.1176/appi.ps.56.12.1600

Bower, P., Garralda, E., Kramer, T., Harrington, R., \& Sibbald, B. (2001). The treatment of child and adolescent mental health problems in primary care: A systematic review. Family Practice, 18, 373-382. doi:10.1093/fampra/18.4.373

Boyd, M., \& Foley, M. (2009). Psychiatric nursing: Contemporary practice (4th ed.). Philadelphia: Wolters Kluwer Health/Lippincott Williams \& Wilkins.

Cardemil, E. V., Reivich, K. J., \& Seligman, M. E. P. (2002). The prevention of depressive symptoms in low-income minority middle school students. Prevention \& Treatment, 5, 1-28. doi:10.1037/1522-3736.5.1.58a

Centers for Disease Control and Prevention National Center for Injury Prevention and Control (2012). Web-based Injury Statistics Query and Reporting System (WISQARS). www.cdc.gov/ncipc/wisqars

Choi, H., \& Gi Park, C. (2006). Understanding adolescent depression in ethnocultural context: Updated with empirical findings. Advances in Nursing Science, 29, E1-E12.

Collins, M. H., Kelch-Oliver, K., Welkom, J. S., Johnson, K., Kottke, M., \& Smith, C. O. (2010). Clinically significant depressive symptoms in African American adolescetn females in an urban reproductive health clinic. Journal of Clinical Psychology in Medical Settings, 17, 175-182. doi:10.1007/s10880-010-9200-9

Copeland, V. C. (2006). Disparities in mental health service utilization among low-income African American adolescents: Closing the gap by enhancing practitioner's competence. Child and Adolescent Social Work Journal, 23, 407-431. doi:10.1007/s10560-006-0061-X

Costello, J., Erkanli, A., \& Angold, A. (2006). Is there an epidemic of child or adolescent depression? Journal of Child Psychology \& Psychiatry, 47, 1263-1271.

Cote, M., Mullins, L., Hartman, V., Hoff, A., Balderson, B., Chaney, J., \& Domek, D. (2003). Psychosocial correlates of health care utilization for children and adolescents with type 1 diabetes mellitus. Children s Health Care, 32, 1-16. doi:10.1207/S15326888CHC3201_1

Frankenfield, D. L., Keyl, P. M., Gielen, A., Wissow, L. S., Werthamer, L., \& Baker, S. P. (2000). Adolescent patients-Healthy or hurting? Missed opportunities to screen for suicide risk in the primary care setting. Archives of Pediatrics and Adolescent Medicine, 154, 162168.

Franko, D., Striegel-Moore, R., Bean, J., Tamer, R., Kraemer, H., Dohm, F., Crawford, P., Schreiber, G., \& Daniels, S. (2005). Psychosocial and health consequences of adolescent depression in black and white young adult women. Health Psychology, 24, 586-593. doi:10.1037/0278-6133.24.6.586

Gallo, J. J., Bogner, H. R., Morales, K. H., \& Ford, D. E. (2005). Patient ethnicity and the identification and active management of depression in late life. Archives of Internal Medicine, 165, 1962-1968. doi:10.1001/archinte.165.17.1962

Gibbs, J. T. (1988). Conceptual, methodological, and sociocultural issues in Black youth suicide: Implications for assessment and early intervention. Suicide \& Life-Threatening Behavior, 18, 73-89.

Goldston, D. B., Molock, S. D., Whitbeck, L. B., Murakami, J. L., Zayas, L. H., \& Hall, G. C. N. (2008). Cultural considerations in adolescent suicide prevention and psychosocial treatment. American Psychologist, 63, 14. doi:10.1037/0003-066X.63.1.14

Hacker, K. A., Myagmarjav, E., Harris, V., Suglia, S. F., Weidner, D., \& Link, D. (2006). Mental health screening in pediatric practice: Factors related to positive screens and the contribution of parental/personal concern. Pediatrics, 118, 1896-1906. doi:10.1542/peds.2006-0026

Hammack, P. L., Richards, M. H., Luo, Z., Edlynn, E. S., \& Roy, K. (2004). Social support factors as moderators of community violence exposure among inner-city African American young adolescents. Journal of Clinical Child and Adolescent Psychology, 33, 450-462. doi:10.1207/s15374424j_cp3303_3

Hamrin, V., \& Magorno, M. (2010). Assessment of adolescents for depression in the pediatric primary care setting. Pediatric nursing, 36, 103-111.

Horowitz, J. L., \& Garber, J. (2006). The prevention of depressive symptoms in children and adolescents: A meta-analytic review. Journal of Consulting and Clinical Psychology, 74, 401-415. doi:10.1037/0022-006X.74.3.401

Husky, M. M., Miller, K., McGuire, L., Flynn, L., \& Olfson, M. (2011). Mental health screening of adolescents in pediatric practice. The Journal of Behavioral Health Services and Research, 38, 159-169. doi:10.1007/s11414-009-9207-X

Johnson, J. G., Harris, E., Spitzer, R. L., \& Williams, J. B. W. (2002). The patient health questionaire for adolescents: Validation of an instrument for the assessment of mental disorders among adolescent primary care patients. Journal of Adolescent Health, 30, 196-204. doi:10.1016/S1054-139X(01)00333-0

Kelleher, K. J., Campo, J. V., \& Gardner, W. P. (2006). Management of pediatric mental disorders in primary care: Where are we now and where are we going? Current Opinion in Pediatrics, 18, 649-653. doi:10.1097/MOP.0b013e3280106a76

Kessler, R. C., Avenevoli, S., \& Ries Merikangas, K. (2001). Mood disorders in children and adolescents: An epidemiologic perspective. Biological Psychiatry, 49, 1002-1014.

doi:10.1016/S0006-3223(01)01129-5

Kessler, R. C., Berglund, P., Demler, O., Jin, R., Koretz, D., Merikangas, K. R., Rush, A. J., Walters, E. E., \& Wang, P. S. (2003). The epidemiology of major depressive disorder. JAMA: The Journal of the American Medical Association, 289, 3095-3105. doi:10.1001/jama.289.23.3095

Kramer, T., Beaudin, C., \& Thrush, C. (2005). Evaluation and treatment of depression (Part I): Benefits for patients, providers, and payors. Disease Management and Health Outcomes, 13, 295-306. doi:10.2165/00115677-200513050-00002

Lindsey, M. A., Joe, S., \& Nebbitt, V. (2010). Family matters: The role of mental health stigma and social support on depressive symptoms and subsequent help seeking among African American boys. Journal of Black Psychology, 36, 458-482. doi:10.1177/0095798409355796

Mazzaferro, K., Murray, P., Ness, R., Bass, D., Tyus, N., \& Cook, R. (2006). Depression, stress, and social support as predictors of highrisk sexual behaviors and STIs in young women. Journal of Adolescent Health, 39, 601-603. doi:10.1016/j.jadohealth.2006.02.004

Molock, S. D., Barksdale, C., Matlin, S., Puri, R., Cammack, N., \& Spann, M. (2007). Qualitative study of suicidality and help-seeking behaviors in African American adolescents. American Journal of Community Psychology, 40, 52-63. doi:10.1007/s10464-007-9122-3

Moses, T. (2009). Self-labeling and its effects among adolescents diagnosed with mental disorders. Social Science \& Medicine, 68, 570-578. doi:10.1016/j.socscimed.2008.11.003

Nebbitt, V. E., \& Lombe, M. (2008). Assessing the moderating effects of depressive symptoms on antisocial behavior among urban youth in public housing. Child and Adolescent Social Work Journal, 25, 409424. doi:10.1007/s10560-008-0134-0

Nimalasuriya, K., Compton, M. T., \& Guillory, V. J. (2009). Screening adults for depression in primary care: A position statement of the American College of Preventive Medicine. Journal of Family Practice, 58, 535-538.

Radloff, L. (1977). The CES-D scale: A self-report depression scale for research in the general population. Applied Psychological Measure- 
ment, 1, 385-401. doi:10.1177/014662167700100306

Radloff, L. (1991). The use of the Center for Epidemiologic Studies Depression Scale in adolescents and young adults. Journal of Youth and Adolescence, 20, 149-166. doi:10.1007/BF01537606

Riolo, S., Nguyen, T., Greden, J., \& King, C. (2005). Prevalence of depression by race/ethnicity: Findings from the National Health and Nutrition Examination Survey III. American Journal of Public Health, 95, 998-1000. doi:10.2105/AJPH.2004.047225

Rudolph, K. D. (2008). Adolescent depression. In I. H. Gotlib, \& C. L. Hammen (Eds.), Handbook of depression (2nd ed., pp. 444-446). New York, NY: The Guilford Press.

Rushton, J., Forcier, M., \& Schectman, R. (2002). Epidemiology of depressive symptoms in the National Longitudinal Study of Adolescent Health. Journal of the American Academy of Child \& Adolescent Psychiatry, 41, 199-205. doi:10.1097/00004583-200202000-00014

Shaffer, A., Forehand, R., \& Kotchick, B. (2002). A longitudinal examination of correlates of depressive symptoms among inner-city African-American children and adolescents. Journal of Child and Family Studies, 11, 151-164. doi:10.1023/A:1015121424404

Stein, R. E. K., Zitner, L. E., \& Jensen, P. S. (2006). Interventions for adolescent depression in primary care. Pediatrics, 118, 669-682. doi:10.1542/peds.2005-2086

Stockdale, S. E., Lagomasino, I. T., Siddique, J., McGuire, T., \& Miranda, J. (2008). Racial and ethnic disparities in detection and treatment of depression and anxiety among psychiatric and primary health care visits, 1995-2005. Medical Care, 46, 668-677. doi:10.1097/MLR.0b013e3181789496

Substance Abuse and Mental Health Services Administration Office of Applied Studies (2008). The NSDUH Report-Major depressive episodes among youths aged 12 to 17 in the United States: 2004 to 2006. Rockville, MD.

US Department of Health and Human Services (2001). Mental health: Culture, race, and ethnicity-A supplement to mental heatlh: A report of the Surgeon General. Rockville, MD: US Department of Health and Human Services, Substance Abuse and Mental Health Services Administration, Center for Mental Health Services

US Department of Health and Human Services (2008). Results from the 2007 National Survey on Drug Use and Health: National findings. (DHHS Publication No. SMA 08-4343, NSDUH Series H-34). Rockville, MD: Substance Abuse and Mental Health Services Administration

US Preventive Services Task Force (2009). Screening and treatment for major depressive disorder in children and adolescents: Clinical
Summary.

http://www.uspreventiveservicestaskforce.org/uspstf09/depression/ch deprsum.htm

Walker, Z., Townsend, J., Oakley, L., Donovan, C., Smith, H., Hurst, Z., Bell, J., \& Marshall, S. (2002). Health promotion for adolescents in primary care: Randomised controlled trial. British Medical Journal, 325, 524-530. doi:10.1136/bmj.325.7363.524

Westwood, M., \& Pinzon, J. (2008). Adolescent male health. Paediatrics \& Child Health, 13, 31-36.

Wight, R. G., Aneshensel, C. S., Botticello, A. L., \& Sepúlveda, J. E. (2005). A multilevel analysis of ethnic variation in depressive symptoms among adolescents in the United States. Social Science \& Medicine, 60, 2073-2084. doi:10.1016/j.socscimed.2004.08.065

Williams, J., Klinepeter, K., Palmes, G., Pulley, A., \& Foy, J. M. (2004). Diagnosis and treatment of behavioral health disorders in pediatric practice. Pediatrics, 114, 601-606. doi:10.1542/peds.2004-0090

Williams, S. B., O'Connor, E. A., Eder, M., \& Whitlock, E. P. (2009). Screening for child and adolescent depression in primary care settings: a systematic evidence review for the US Preventive Services Task Force. Pediatrics, 123, e716-e736. doi:10.1542/peds.2008-2415

Winter, L. B., Steer, R. A., Jones-Hicks, L., \& Beck, A. T. (1999). Screening for major depression disorders in adolescent medical outpatients with the Beck Depression Inventory for Primary Care. Journal of Adolescent Health, 24, 389-394. doi:10.1016/S1054-139X(98)00135-9

Wu, P., Hoven, C. W., Cohen, P., Liu, X., Moore, R. E., Tiet, Q., Okezie, N., Wicks, J., \& Bird, H. R. (2001). Factors associated with use of mental health services for depression by children and adolescents. Psychiatric Services, 52, 189-195. doi:10.1176/appi.ps.52.2.189

Zuckerbrot, R. A., Cheung, A. H., Jensen, P. S., Stein, R. E. K., \& Laraque, D. (2007). Guidelines for Adolescent Depression in Primary Care (GLAD-PC): I. Identification, assessment, and initial management. Pediatrics, 120, e1299-e1312. doi:10.1542/peds.2007-1144

Zuckerbrot, R. A., \& Jensen, P. S. (2006). Improving recognition of adolescent depression in primary care. Archives of Pediatrics and Adolescent Medicine, 160, 694-704. doi:10.1001/archpedi.160.7.694

Zuckerbrot, R. A., Maxon, L., Pagar, D., Davies, M., Fisher, P. W., \& Shaffer, D. (2007). Adolescent depression screening in primary care: Feasibility and acceptability. Pediatrics, 119, 101-108.

doi:10.1542/peds.2005-2965 